Copyright by the Optical Society of America. Ron Pieper, Deborah Koslover, and Ting-Chung Poon, "Exact solution for four-order acousto-optic Bragg diffraction with arbitrary initial conditions," Appl. Opt. 48, C141-C150 (2009); doi: $10.1364 / \mathrm{AO} .48 .00 \mathrm{C} 141$

\title{
Exact solution for four-order acousto-optic Bragg diffraction with arbitrary initial conditions
}

\author{
Ron Pieper, ${ }^{1, *}$ Deborah Koslover, ${ }^{2}$ and Ting-Chung Poon ${ }^{3}$ \\ 'Department of Electrical Engineering, University of Texas at Tyler, 3900 University Boulevard, Tyler, Texas 75799, USA \\ ${ }^{2}$ Department of Mathematics, University of Texas at Tyler, 3900 University Boulevard, Tyler, Texas 75799, USA \\ ${ }^{3}$ Department of Electrical and Computer Engineering, Virginia Tech, Blacksburg, Virginia 24061, USA \\ *Corresponding author: rpieper@uttyler.edu
}

Received 13 August 2008; revised 20 November 2008; accepted 5 December 2008; posted 9 December 2008 (Doc. ID 100081); published 26 January 2009

\begin{abstract}
An exact solution to the four-order acousto-optic (AO) Bragg diffraction problem with arbitrary initial conditions compatible with exact Bragg angle incident light is developed. The solution, obtained by solving a 4th-order differential equation, is formalized into a transition matrix operator predicting diffracted light orders at the exit of the AO cell in terms of the same diffracted light orders at the entrance. It is shown that the transition matrix is unitary and that this unitary matrix condition is sufficient to guarantee energy conservation. A comparison of analytical solutions with numerical predictions validates the formalism. Although not directly related to the approach used to obtain the solution, it was discovered that all four generated eigenvalues from the four-order AO differential matrix operator are expressed simply in terms of Euclid's Divine Proportion. @ 2009 Optical Society of America OCIS codes: $\quad 070.1060,000.4430$.
\end{abstract}

\section{Introduction}

Since the observations of Debye and Sears [1] on the interactions of light and sound, there has been continued interest in developing reliable criteria for determining the conditions in which only one order of light is significantly diffracted. General background review material for $\mathrm{AO}$ developments can be found in Ref. [2]. Notwithstanding the power of numerical methods to provide physical solutions for mathematically intractable specified designs $[3,4]$, an analytic solution, if available, is usually preferred. In the early 1980s, an approximate solution involving four diffracted orders was developed by Poon and Korpel based on Feynman diagram techniques [5]. This solution took the form of an infinite series in powers of $1 / Q$, where $Q$ is the Klein-Cook parameter and practical computation requires the truncation of the series. Around the same time, Poon worked out an exact solution involving four diffracted orders [6], assum-

0003-6935/09/07C141-10\$15.00/0

(C) 2009 Optical Society of America ing $100 \%$ of the energy present in the 0 th order and incident at the exact Bragg angle. This four-order solution was derived from simultaneous analysis of two 2nd-order differential equations and involved boundary conditions related to both initial 0th-order and-1-order field values as well as their corresponding space derivatives. The validity of the solution was checked by confirming that it met the energy conservation rule. More recently, Blomme and Leroy [7] worked out an exact four-order solution that was applicable to arbitrary angle of incidence, but again was derived only with boundary conditions in which $100 \%$ of the optical power was initialized in the 0 th order. The development of a four-order solution for arbitrary boundary conditions would be necessary to establish the equivalent of a four-order matrix formalism comparable to the two-order matrix formalism [8] previously studied. The physical and mathematical model employed in this work is based on Korpel's 1979 paper [9] on two-dimensional strong interaction of light and sound. The proposed fourorder solution developed here is for arbitrary initial conditions and exact Bragg angle of incidence. The 
solution can be conveniently programmed for a variety of boundary conditions and it compares favorably with a normalized numerical analysis solution using Euler's method. Due to space limitations, the details of the numerical analysis scheme are not presented, but they can be reviewed elsewhere $[3,4,8]$. The derived four-order solution is shown to satisfy energy conservation. This requires that the adjoint of the matrix form of the solution is equal to the inverse of the matrix solution, i.e., unitary [10]. Although not directly tied into the mathematics of the approach taken to find the four-order solution, our results unveiled that the Golden Ratio, also known as the Divine Proportion [11], found in specimens and examples from art, biology, architecture, and mathematics, appears in the mathematics of the four-order AO problem considered here. To avoid ambiguous notation we follow the convention that vectors are typeset in boldface; scalars and matrices are both in lightface but can be readily distinguished by context.

\section{Background}

To keep this paper self-contained and establish notation, we briefly review the principles of acoustooptics. Table 1 lists and defines various fundamental parameters appearing in this discussion. The uppercase parameters have been reserved for acoustic parameters and the lowercase for optical parameters. The following wave relationships exist between wavenumbers $K, k$; wavelengths $\Lambda, \lambda$; and radian frequencies $\Omega, \omega$. We have

$$
\begin{gathered}
K=\frac{2 \pi}{\Lambda}=\frac{\Omega}{V_{s}}, \\
k=\frac{2 \pi}{\lambda}=\frac{\omega}{c} .
\end{gathered}
$$

Note that Table 1 identifies standard assumptions made on the relative scale for typical values of optical versus acoustic parameters. The conservation of momentum diagram [2] can be used to predict the Bragg angle which, as seen in Fig. 1, is exactly one half of the angle between the adjacent diffracted orders, where the Bragg angle $\phi_{B}$ is given by

$$
\phi_{B}=\sin ^{-1}\left(\frac{K}{2 k}\right) \approx K /(2 k)=\Omega /\left(2 k V_{s}\right) .
$$

The diffraction angles $\phi_{n}$ for various diffracted orders can be expressed in terms of a 0th-order angle $\phi_{0}$ defined between horizontal reference $(z$-axis) and the

Table 1. Fundamental Parameters (After Pieper et al. [3]

\begin{tabular}{lccc}
\hline Description & Acoustic & Relation & Optical \\
\hline Wavelength & $\Lambda$ & $\gg$ & $\lambda$ \\
Wave speed & $V_{s}$ & $\ll$ & $c$ \\
Radian frequency & $\Omega$ & $\ll$ & $\omega$ \\
Wavenumber & $K$ & $\ll$ & $k$ \\
\hline
\end{tabular}

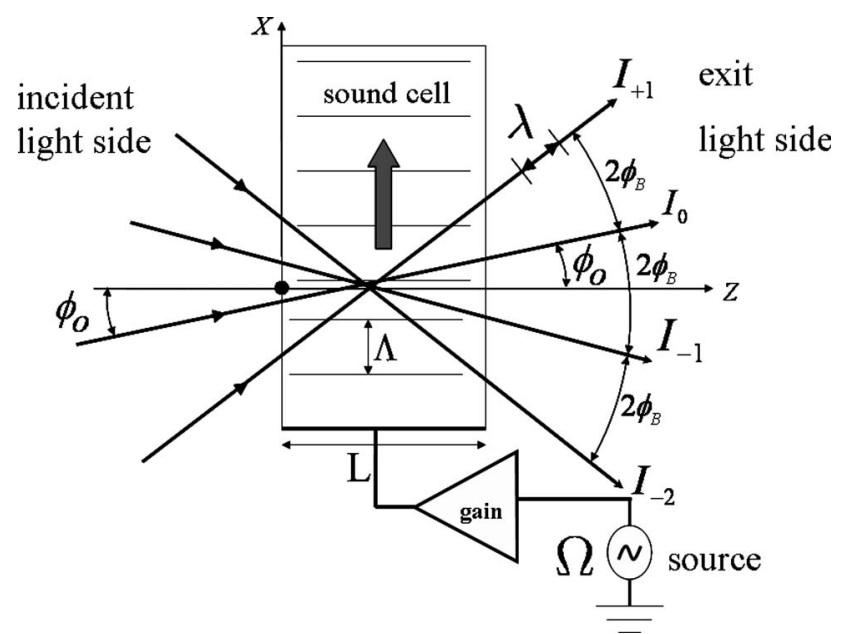

Fig. 1. Selected physical parameters and possible incident light rays identified on incident light side. $L$ is the length of the acoustic transducer along the $z$ direction.

0th-order light ray trajectory, as seen in Fig. 1 , and is given by

$$
\phi_{n}=\phi_{0}+2 n \phi_{B}
$$

where $-2 \leq n \leq 1$ for the problem involving four diffracted orders.

For operation in the Bragg regime [2], more than $90 \%$ of the zeroth order incident power, where $\phi_{\text {inc }}=\phi_{0}$, is diverted into the first diffracted order of the spectrum, i.e., for $n=-1$. This requires that two conditions are satisfied. First, a condition of exact Bragg diffraction,

$$
\Delta \phi \equiv \phi_{0}-\phi_{B} \approx 0 \quad \text { (Bragg regime condition), }
$$

and second,

$$
Q \equiv 2 \pi L \frac{\lambda}{\Lambda^{2}}>2 \pi \quad \text { (Bragg regime condition) }
$$

where $Q$ is the Klein-Cook parameter [12]. Although not always mentioned, if the sound field amplitude is too high then spurious orders, other than 0 and -1 , are generated from the acoustic sidelobes [3].

A formalism for strong AO interaction proposed in the late 1970s by Korpel [9] is introduced in order to proceed with the analysis. The following differential equation formalism couples electric field phasors between neighboring diffracted orders $\psi_{n}, \psi_{n-1}$, and $\psi_{n+1}$ :

$$
\Delta \psi_{n}(z)=-j a\left(S_{n-1}^{+}(z) \psi_{n-1}(z)+S_{n+1}^{-}(z) \psi_{n+1}(z)\right) \Delta z .
$$

The electric field phasors are defined according to an engineering convention with Doppler shifted harmonics $\omega_{n}=\omega+n \Omega$ and phase referenced back to the origin at cell entrance $z=0$. Furthermore, in Eq. (5), we have 


$$
a=k C / 4
$$

where $C$ is a constant that gauges the strain-optic sensitivity. A time-dependent acoustic field $S(\rho, t)$ introduces compression and rarefaction on index of refraction $n$, which changes according to $\delta n(\rho, t)=$ $\mathrm{CS}(\rho, t)$. Note that $\rho=(x, z)$ identifies the coordinates for a point in the sound field, as shown in Fig. 2. The sound field is assumed to be time harmonic at sound frequency $\Omega$, and accordingly the sound phasor $\tilde{S}(\rho) \equiv \tilde{S}(x, z)$ can be defined via the engineering convention, i.e., $S(\rho, t)=\operatorname{Re}\{\tilde{S}(\rho) \exp (j \Omega t)\}$. The sound coefficients $S_{n-1}^{+}(z)$ and $S_{n+1}^{-}(z)$ in Eq. (5) are then obtained after defining the $x$ component of the bisectors for the diffracted orders:

$$
x_{n}^{ \pm} \equiv z \tan \left(\phi_{n} \pm \phi_{B}\right) \approx z\left(\phi_{n} \pm \phi_{B}\right) .
$$

These bisectors are known as Bragg lines [2,9] and appear as dashed lines in Fig. 2. The solid lines in Fig. 2 represent the trajectories for the diffracted orders. Approximation (7) is valid, assuming small angles for all diffracted orders, which gives

$$
\begin{gathered}
S_{n-1}^{+}(z) \equiv \tilde{S}\left(z, x_{n-1}^{+}\right) \approx \tilde{S}\left(z, z\left(\phi_{n-1}+\phi_{B}\right)\right), \\
S_{n+1}^{-}(z) \equiv \tilde{S}^{*}\left(z, x_{n+1}^{-}\right) \approx \tilde{S}^{*}\left(z, z\left(\phi_{n+1}-\phi_{B}\right)\right),
\end{gathered}
$$

where the superscript $*$ indicates the complex conjugate.

For the bounded sound column plane-wave model, the dependence of the sound phasor on the $(x, z)$ variable is taken to be separable according to

$$
\tilde{S}(z, x)= \begin{cases}|\tilde{S}(z)| \exp (-j K x+j \theta(z)) & z \text { in }[0, L] \\ 0 & \text { otherwise }\end{cases}
$$

where $\theta(z)$ in an ad hoc manner accounts for any special design related $z$-dependent phase shift along the transducer. This would, for example, be nonzero in

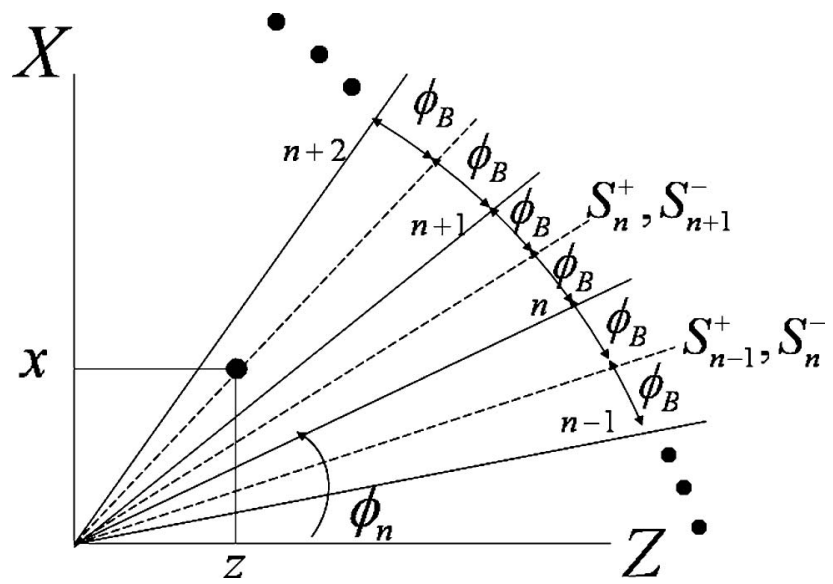

Fig. 2. General AO interaction diagram showing (dashed) Bragg lines (after Pieper et al. []] ). the case that the design of the transducer employs phased-array techniques [4]. After combining Eqs. (י) $)$ ()), and (ㅁ), we have

$$
S_{n-1}^{+}(z)=\tilde{S}(z) \exp \left(-j K z\left(\phi_{0}+(2 n-1) \phi_{B}\right)+j \theta(z)\right)
$$

$$
S_{n+1}^{-}(z)=\tilde{S}^{*}(z) \exp \left(+j K z\left(\phi_{0}+(2 n+1) \phi_{B}\right)-j \theta(z)\right)
$$

The AO transducer manufacturer will typically provide specifications on the operating power limits and a center frequency of operation $\Omega_{c}$. We will not repeat the algebraic detail that is described elsewhere $[3,4,8]$. The existence of a center frequency permits a normalization in many of the predominant $\mathrm{AO}$ parameters, including frequency, Bragg angle, and incident angle, as outlined in Table 2 . In addition, as described in Table 2 , the space coordinate $z$ can be included in the normalization scheme. In the context of the normalization described, the sound coefficients in Eq. (10) can be recast in the following forms:

$$
\begin{aligned}
S_{n-1}^{+}(\xi)= & \tilde{S}(\xi) \exp \left(-j \frac{1}{2} Q_{c} \bar{\Omega}^{2} \xi\left(\overline{\frac{\phi_{0}}{\bar{\Omega}}}+(2 n-1)\right)\right. \\
& +j \theta(\xi))
\end{aligned}
$$

$$
\begin{aligned}
& S_{n+1}^{-}(\xi)=\tilde{S}^{*}(\xi) \exp \left(+j \frac{1}{2} Q_{c} \bar{\Omega}^{2} \xi\left(\frac{\overline{\phi_{0}}}{\bar{\Omega}}+(2 n+1)\right)\right. \\
& -j \theta(\xi))
\end{aligned}
$$

and the original coupled equation (5) is given in a normalized form by

$$
\Delta \psi_{n}(\xi)=-j a L\left(S_{n-1}^{+}(\xi) \psi_{n-1}(\xi)+S_{n+1}^{-}(\xi) \psi_{n+1}(\xi)\right) \Delta \xi
$$

Special cases considered in this work are identified according to the following conditions:

Table 2. Normalized Parameters ${ }^{a}$

\begin{tabular}{lc}
\hline Normalized Variable & Defining Relation \\
\hline$z$ coordinate & $\xi \equiv z / L$ \\
Acoustic frequency & $\bar{\Omega} \equiv \Omega / \Omega_{c}$ \\
Incident angle of light & $\overline{\phi_{o}} \equiv \phi_{o} / \phi_{B c}$ \\
Klein-Cook parameter & $Q_{c}=L K_{c}^{2} / k$ \\
\hline
\end{tabular}

${ }^{a}$ Subscript $c$ indicates evaluation at the center frequency; after Pieper et al. [3]]. 
$\theta(\xi)=0 \quad$ (no design phase shift)

$S(\xi)=|\tilde{S}|$ (uniform sound field),

$\bar{\Omega}=1 \quad$ (operating at the center frequency),

$\overline{\phi_{0}}=1 \quad($ exact Bragg condition $\Delta \phi=0)$.

Backsubstitution of Eqs. (13) will lead to a special case for Eq. (11) as follows (see Section 4-2.5 of Ref. [2]):

$$
\begin{aligned}
& S_{n-1}^{+}(\xi) /|\tilde{S}|=\exp \left(-j n Q_{c} \xi\right), \\
& S_{n+1}^{-}(\xi) /|\tilde{S}|=\exp \left(+j(n+1) Q_{c} \xi\right) .
\end{aligned}
$$

And following subsequent simplification of Eq. (12) using Eqs. (14) leads to

$$
\Delta \psi_{n}(\xi)=-j \alpha\left(e^{-j n Q_{c} \xi} \psi_{n-1}(\xi)+e^{j(n+1) Q_{c} \xi} \psi_{n+1}(\xi)\right) \Delta \xi
$$

where the sound factor

$$
\alpha \equiv \alpha|\tilde{S}| L
$$

is typically selected to be $\pi / 2$ in order to achieve a maximum in the efficiency of diffraction from the 0 th order into the -1 st order [2]. For the four orders $\Psi^{T}(\xi)=\left(\psi_{+1}(\xi), \psi_{0}(\xi), \psi_{-1}(\xi), \bar{\psi}_{-2}(\xi)\right)$ the coupled differential equation (15) can be placed into matrix form:

$$
\frac{d \Psi(\xi)}{d \xi}=-j \alpha A(\xi) \Psi(\xi)
$$

where

$A=\left(\begin{array}{cccc}0 & \exp \left(-j Q_{c} \xi\right) & 0 & 0 \\ \exp \left(+j Q_{c} \xi\right) & 0 & 1 & 0 \\ 0 & 1 & 0 & \exp \left(+j Q_{c} \xi\right) \\ 0 & 0 & \exp \left(-j Q_{c} \xi\right) & 0\end{array}\right)$

The special case here leads to a Hermitian operator matrix (18), and therefore it has four real eigenvalues [10]. The interesting observation is that the eigenvalues are either equal in magnitude to the Divine Proportion [11] or equal in magnitude to the reciprocal of this constant; see Appendix A for details.

\section{Solution for the Four-Order Problem}

Through a lengthy substitution process starting from Eq. (17) it can be shown that the solution for the -2 diffracted order satisfies a 4th-order linear homogeneous constant-coefficient differential equation as follows:

$$
\begin{aligned}
& \frac{d^{4} \psi_{-2}}{d \xi^{4}}+2 j Q_{c} \frac{d^{3} \psi_{-2}}{d \xi^{3}}+\left(3 \alpha^{2}-Q_{c}^{2}\right) \frac{d^{2} \psi_{-2}}{d \xi^{2}} \\
& +2 j Q_{c} \alpha^{2} \frac{d \psi_{-2}}{d \xi}+\alpha^{4} \psi_{-2}=0 .
\end{aligned}
$$

The salient features of this derivation are retained in Appendix B. The form for Eq. (19) suggests four independent solutions of the form $\psi_{-2} \doteq \exp (j r \xi)$. Substitution back into Eq. (19) leads to a quartic polynomial in root $r$ :

$$
r^{4}+2 Q_{c} r^{3}+\left(Q_{c}^{2}-3 \alpha^{2}\right) r^{2}-2 Q_{c} \alpha^{2} r+\alpha^{4}=0,
$$

and the roots obtained are

$$
r_{1}=-\frac{1}{2}\left(Q_{c}+\alpha+\left(Q_{c}^{2}+2 Q_{c} \alpha+5 \alpha^{2}\right)^{1 / 2}\right),
$$

$$
\begin{aligned}
& r_{2}=-\frac{1}{2}\left(Q_{c}+\alpha-\left(Q_{c}^{2}+2 Q_{c} \alpha+5 \alpha^{2}\right)^{1 / 2}\right), \\
& r_{3}=-\frac{1}{2}\left(Q_{c}-\alpha+\left(Q_{c}^{2}-2 Q_{c} \alpha+5 \alpha^{2}\right)^{1 / 2}\right), \\
& r_{4}=-\frac{1}{2}\left(Q_{c}-\alpha-\left(Q_{c}^{2}-2 Q_{c} \alpha+5 \alpha^{2}\right)^{1 / 2}\right),
\end{aligned}
$$

which are consistent with results previously found [6]. Note that it can be asserted from observation that all the roots are real as seen from

$$
Q_{c}^{2} \pm 2 Q_{c} \alpha+5 \alpha^{2}=\left(Q_{c} \pm \alpha\right)^{2}+4 \alpha^{2} .
$$

From a substitution of Eq. (22) into Eqs. (21) two roots $\left(r_{2}\right.$ and $\left.r_{4}\right)$ are identified to be negative and the other two roots $\left(r_{1}\right.$ and $\left.r_{3}\right)$ are identified as positive. The general solution to Eq. (19) is a superposition of the four homogeneous solutions,

$$
\begin{aligned}
\psi_{-2}(\xi)= & A \exp \left(j r_{1} \xi\right)+B \exp \left(j r_{2} \xi\right)+C \exp \left(j r_{3} \xi\right) \\
& +D \exp \left(j r_{4} \xi\right)
\end{aligned}
$$

and the coupling back to the other orders can be shown to lead to a consistent solution form for all orders given as follows: 


$$
\begin{aligned}
\psi_{+1}(\xi)= & A \exp \left(j r_{1} \xi\right)+B \exp \left(j r_{2} \xi\right)-C \exp \left(j r_{3} \xi\right) \\
& -D \exp \left(j r_{4} \xi\right)
\end{aligned}
$$

$$
\begin{aligned}
\psi_{0}(\xi)= & -\frac{1}{\alpha}\left\{r_{1} A \exp \left[j\left(r_{1}+Q_{c}\right) \xi\right]\right. \\
& +r_{1} B \exp \left[j\left(r_{2}+Q_{c}\right) \xi\right]-r_{1} C \exp \left[j\left(r_{3}+Q_{c}\right) \xi\right] \\
& \left.-r_{1} D \exp \left[j\left(r_{4}+Q_{c}\right) \xi\right]\right\},
\end{aligned}
$$

$$
\begin{aligned}
\psi_{-1}(\xi)= & -\frac{1}{\alpha}\left\{r_{1} A \exp \left[j\left(r_{1}+Q_{c}\right) \xi\right]\right. \\
& +r_{1} B \exp \left[j\left(r_{2}+Q_{c}\right) \xi\right]+r_{1} C \exp \left[j\left(r_{3}+Q_{c}\right) \xi\right] \\
& \left.+r_{1} D \exp \left[j\left(r_{4}+Q_{c}\right) \xi\right]\right\},
\end{aligned}
$$

$$
\begin{aligned}
\psi_{-2}(\xi)= & A \exp \left(j r_{1} \xi\right)+B \exp \left(j r_{2} \xi\right)+C \exp \left(j r_{3} \xi\right) \\
& +D \exp \left(j r_{4} \xi\right)
\end{aligned}
$$

An evaluation of the special case of Eqs. (24) at $\xi=$ 0 links initial conditions from the left-hand side of Eqs. (24) to unknown coefficients $\{A, B, C, D\}$ appearing on the right-hand side of Eqs. (24). Solving for $\{A, B, C, D\}$ with $\xi=0$ leads to a set of prediction rules for the unknown coefficients:

$$
\begin{aligned}
A= & \frac{1}{2\left(r_{2}-r_{1}\right)}\left(r_{2} \psi_{+1}(0)+\alpha \psi_{0}(0)+\alpha \psi_{-1}(0)\right. \\
& \left.+r_{2} \psi_{-2}(0)\right) \\
B= & \frac{1}{2\left(r_{2}-r_{1}\right)}\left(-r_{1} \psi_{+1}(0)-\alpha \psi_{0}(0)-\alpha \psi_{-1}(0)\right. \\
& \left.-r_{1} \psi_{-2}(0)\right) \\
C= & \frac{1}{2\left(r_{3}-r_{4}\right)}\left(r_{4} \psi_{+1}(0)+\alpha \psi_{0}(0)-\alpha \psi_{-1}(0)\right. \\
& \left.-r_{4} \psi_{-2}(0)\right), \\
D= & \frac{1}{2\left(r_{3}-r_{4}\right)}\left(-r_{3} \psi_{+1}(0)-\alpha \psi_{0}(0)+\alpha \psi_{-1}(0)\right. \\
& \left.+r_{3} \psi_{-2}(0)\right),
\end{aligned}
$$

Substituting Eqs. (25) into Eqs. (24) we obtain

$$
\begin{aligned}
\psi_{+1}(\xi)= & \frac{1}{2}\left[(\sigma+\tau) \psi_{+1}(0)+\alpha(\mu-\rho) \psi_{0}(0)\right. \\
& \left.+\alpha(\mu+\rho) \psi_{-1}(0)+(\sigma-\tau) \psi_{-2}(0)\right],
\end{aligned}
$$

$$
\begin{aligned}
\psi_{0}(\xi)= & \frac{1}{2} \exp \left(j Q_{c} \xi\right)\left[\alpha(\mu-\rho) \psi_{+1}(0)+(\theta+\gamma) \psi_{0}(0)\right. \\
& \left.+(\theta-\gamma) \psi_{-1}(0)+\alpha(\mu+\rho) \psi_{-2}(0)\right]
\end{aligned}
$$

$$
\begin{aligned}
\psi_{-1}(\xi)= & \frac{1}{2} \exp \left(j Q_{c} \xi\right)\left[\alpha(\mu+\rho) \psi_{+1}(0)+(\theta-\gamma) \psi_{0}(0)\right. \\
& \left.+(\theta+\gamma) \psi_{-1}(0)+\alpha(\mu-\rho) \psi_{-2}(0)\right]
\end{aligned}
$$

$$
\begin{aligned}
\psi_{-2}(\xi)= & \frac{1}{2}\left[(\sigma-\tau) \psi_{+1}(0)+\alpha(\mu+\rho) \psi_{0}(0)\right. \\
& \left.+\alpha(\mu-\rho) \psi_{-1}(0)+(\sigma+\tau) \psi_{-2}(0)\right],
\end{aligned}
$$

where to simplify notation we have introduced

$$
\mu \equiv \frac{\exp \left(j r_{1} \xi\right)-\exp \left(j r_{2} \xi\right)}{r_{2}-r_{1}}
$$

$$
\rho \equiv \frac{\exp \left(j r_{3} \xi\right)-\exp \left(j r_{4} \xi\right)}{r_{3}-r_{4}}
$$

$$
\sigma \equiv \frac{r_{2} \exp \left(j r_{1} \xi\right)-r_{1} \exp \left(j r_{2} \xi\right)}{r_{2}-r_{1}}
$$

$$
\tau \equiv \frac{-r_{4} \exp \left(j r_{3} \xi\right)+r_{3} \exp \left(j r_{4} \xi\right)}{r_{3}-r_{4}}
$$

$$
\theta \equiv \frac{-r_{1} \exp \left(j r_{1} \xi\right)+r_{2} \exp \left(j r_{2} \xi\right)}{r_{2}-r_{1}}
$$

$$
\gamma \equiv \frac{r_{3} \exp \left(j r_{3} \xi\right)-r_{4} \exp \left(j r_{4} \xi\right)}{r_{3}-r_{4}}
$$

Equations (26) can be written more compactly in matrix form as

$$
\Psi(\xi)=G(\xi) \Psi(0),
$$

where

$$
\Psi(\xi) \equiv\left(\begin{array}{c}
\psi_{+1}(\xi) \\
\psi_{0}(\xi) \\
\psi_{-1}(\xi) \\
\psi_{-2}(\xi)
\end{array}\right)
$$




$$
G(\xi) \equiv \frac{1}{2}\left[\begin{array}{cccc}
\sigma+\tau & \alpha(\mu-\rho) & \alpha(\mu+\rho) & \sigma-\tau \\
\alpha(\mu-\rho) \exp \left(j Q_{c} \xi\right) & (\theta+\gamma) \exp \left(j Q_{c} \xi\right) & (\theta-\gamma) \exp \left(j Q_{c} \xi\right) & \alpha(\mu+\rho) \exp \left(j Q_{c} \xi\right) \\
\alpha(\mu+\rho) \exp \left(j Q_{c} \xi\right) & (\theta-\gamma) \operatorname{exo}\left(j Q_{c} \xi\right) & (\theta+\gamma) \exp \left(j Q_{c} \xi\right) & \alpha(\mu-\rho) \exp \left(j Q_{c} \xi\right) \\
\sigma-\tau & \alpha(\mu+\rho) & \alpha(\mu-\rho) & \sigma+\tau
\end{array}\right]
$$

It is important to note that $G(\xi)$ has the following property:

$$
G\left(G^{T}\right)^{*}=G G^{\dagger}=G^{\dagger} G=I,
$$

where $I$ is the identity matrix. The adjoint operator, also known as the Hermitian conjugate, of a tensor quantity $M$ is designated $M^{\dagger}$ and is defined [10] by two specific order-independent successive operations, i.e., complex conjugation-and transpose. As seen from Eq. (31) the adjoint of the transition matrix $G$ is the matrix inverse or more succinctly $G$ is unitary [13]. In Section 4 we demonstrate that the unitary property, applicable to the AO transition matrix, for any number of orders considered, is sufficient to guarantee the physical property of energy conservation.

To illustrate the implementation of Eq. (30), we consider the following cases with identified initial conditions:

$$
\begin{aligned}
\Psi^{T}(0) \equiv & (0,1,0,0) \Rightarrow \Psi^{T}(\xi)=\frac{1}{2}(\alpha(\mu-\rho),(\theta+\gamma) \\
& \left.\times \exp \left(j Q_{c} \xi\right),(\theta-\gamma) \exp \left(j Q_{c} \xi\right), \alpha(\mu+\rho)\right),
\end{aligned}
$$

$$
\begin{aligned}
\Psi^{T}(0) \equiv & (0,0,1,0) \Rightarrow \Psi^{T}(\xi)=\frac{1}{2}(\alpha(\mu+\rho),(\theta-\gamma) \\
& \left.\times \exp \left(j Q_{c} \xi\right),(\theta+\gamma) \exp \left(j Q_{c} \xi\right), \alpha(\mu-\rho)\right),
\end{aligned}
$$

$$
\begin{array}{r}
\Psi^{T}(0) \equiv(1,0,0,0) \Rightarrow \Psi^{T}(\xi)=\frac{1}{2}(\sigma+\tau, \alpha(\mu-\rho) \\
\left.\times \exp \left(j Q_{c} \xi\right), \alpha(\mu+\rho) \exp \left(j Q_{c} \xi\right), \sigma-\tau\right),
\end{array}
$$

$$
\begin{aligned}
\Psi^{T}(0) \equiv & (0,0,0,1) \Rightarrow \Psi^{T}(\xi)=\frac{1}{2}(\sigma-\tau, \alpha(\mu+\rho) \\
& \left.\times \exp \left(j Q_{c} \xi\right), \alpha(\mu-\rho) \exp \left(j Q_{c} \xi\right), \sigma+\tau\right) .(320
\end{aligned}
$$

Each of the four conditions represents $100 \%$ of the energy in one of the four rays incident on the sound cell from the left as shown in Fig. 1.

\section{Energy Conservation for Unitary Transition Matrices}

Starting from a row vector representation for the solution

$$
\Psi^{T}(\xi) \equiv\left(\psi_{+1}(\xi), \psi_{0}(\xi), \psi_{-1}(\xi), \psi_{-2}(\xi)\right),
$$

the field energy is the sum of the magnitude squared of the incoherently [2] related orders:

$$
\Psi^{\dagger}(\xi) \cdot \Psi(\xi)=\sum_{i=+1}^{-2}\left|\psi_{i}(\xi)\right|^{2}
$$

The first term on the left-hand side of Eq. (34) can be represented in terms of the adjoint operator of the transition matrix $G(\xi)$. After rearrangement of terms with reference to Eq. (28), we have

$$
\Psi^{\dagger}(\xi)=(G(\xi) \cdot \Psi(0))^{\dagger}=\Psi^{\dagger}(0) \cdot G^{\dagger}(\xi) .
$$

From the matrix property involving identity matrix $\mathbf{I}$ and that for arbitrary vector $\mathbf{v}$

$$
\mathbf{v}^{\dagger} \cdot I \cdot \mathbf{v}=\mathbf{v}^{\dagger} \cdot \mathbf{v}
$$

combined with Eqs. (31) and (35) and the left-hand side of Eq. (34), we have

$$
\Psi^{\dagger}(\xi) \cdot \Psi(\xi)=\Psi^{\dagger}(0) \cdot G^{\dagger}(\xi) \cdot G(\xi) \cdot \Psi(0)=\Psi^{\dagger}(0) \cdot \Psi(0) .
$$

Or equivalently from Eq. (34), we have

$$
\sum_{i=+1}^{-2}\left|\psi_{i}(\xi)\right|^{2}=\sum_{i=+1}^{-2}\left|\psi_{i}(0)\right|^{2},
$$

which completes the assertion that the solution is consistent with energy conservation. As a disclaimer, the specific limits on the number of orders could be generalized, and therefore, it can be concluded that the property that a transition matrix being unitary will guarantee AO energy conversion, which can be extended to any number of orders. Because of the simplicity of the conservation rule in Eq. (38), it is convenient to check on the accuracy of $\mathrm{AO}$ numerical tests.

\section{Comments on Limits in Applicability for the Four- Order Solution}

From physical considerations, the derived four-order solution is expected to be an inaccurate model when either (or both) of the following happen. First, if the sound cell becomes too narrow the problem resides more accurately in the Raman-Nath regime, and more than four orders can be argued to be significant. Second, in the case that the sound strength is made 
arbitrarily high, even weakly coupled orders, beyond those included in the four-order formalism, become consequential.

The four-order solution developed, Eqs. (28) and (30), is not immediately applicable as a cell-to-cell matrix operator as discussed for two orders in prior work [8] because the fields predicted at the cell exit are referenced back to the cell entrance $(z=0)$ and not yet referenced to the cell exit $(z=L)$. Furthermore, the solution is limited to exact Bragg angle of incidence conditions in the zeroth order $\left(\phi_{0}=\right.$ $\left.\phi_{B c}\right)$. With this restriction for single cell analysis, the angles of the diffracted orders are consistent with rule (3) for any of the incident side conditions shown in Fig. $\underline{1}$ and tested in the examples for Section $\underline{6}$.

\section{Examples}

To validate the analytical solution, Eqs. (28) and (30), a number of example conditions are considered. The corresponding four-order numerical solutions were generated directly from Eqs. (14) and (15) for direct convenient comparison. A variety of incident light cases, Eqs. (32), were tested with the following combination of typical values for the two parameters: sound strength factor $\alpha=\pi / 2$ and Klein-Cook parameter $Q=2 \pi$. In all cases, normalized to unity, the intensity $I_{i}=\left|\psi_{i}(\xi)\right|^{2}$ for the various orders is plotted. As a check for both the numerical and the analytical solutions, the total intensity,

$$
I_{T}=I_{+1}+I_{0}+I_{-1}+I_{-2}=\sum_{i=+1}^{-2}\left|\psi_{i}(\xi)\right|^{2},
$$

is also computed. In Figs. 3-6 the solid line data identify the numerical predictions from Eqs. (14) and (15); the data using the analytic solution, Eqs. (28) and (30), produced the diffraction-order-specific symbols.

Figure 3 shows the numerical and analytical $\xi$ dependent predicted results when $100 \%$ of the incident light is in the 0th order [see Eq. (32a)]. As seen at the right-hand side of the plot, approximately $90 \%$ of the power is transferred to the -1 order via phonon emission and related photon frequency downconversion [2]. Relatively low percentages remain in the other orders. Agreement between numerical and analytic solutions is excellent with a confirming check shown for the total intensities according to Eq. (39).

Figure 4 shows numerical and analytical predicted results when $100 \%$ of the incident light is in the -1 order [see Eq. (32b)]. It can be observed that the curves both numerically and analytically appear shapewise identical to those observed in Fig. 3, however, the orders have been swapped. Specifically

$$
I_{0} \rightleftarrows I_{-1}, \quad I_{+1} \rightleftarrows I_{-2} .
$$

From a physical viewpoint this provides a validation of the accuracy of the solution. It is known that the Bragg conservation of momentum triangle engenders a fundamental symmetry in the phonon-

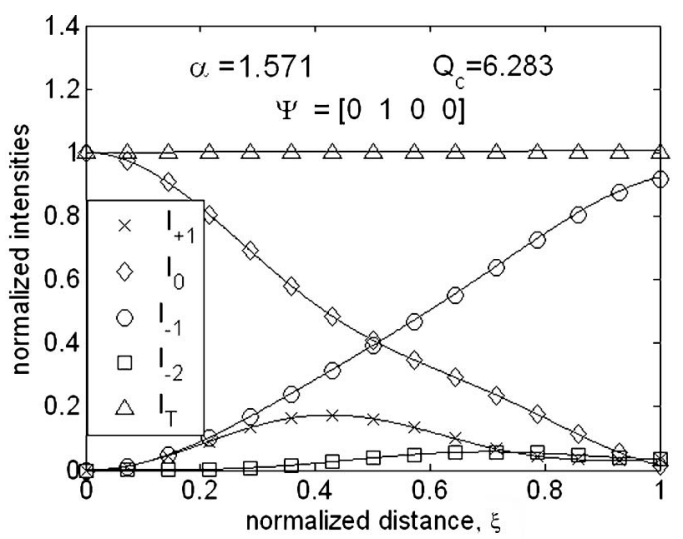

Fig. 3. Comparison for four-order numerical and analytical solutions; all power is initially in the 0 order.

assisted transfer of energy between orders [2]. Figure $\underline{4}$ is then explained in terms of a sound phonon absorbed with a corresponding photon frequency upshift.

Figure 5 shows the numerical and analytical predicted results when $100 \%$ of the incident light is in the +1 order [see Eq. (32c)]. Both numerical and analytical results as before are in complete agreement. The case of $100 \%$ of the light in the -2 order [see Eq. (32d)], was also tested and compared. Curves were again shapewise identical to those in Fig. 5 with a repeated pattern [refer to Eq. (40)]. The plots show that not much power at the end of the cell is coupled out of the $\psi_{+1}$ order. A qualitative observation is that the angular spectrum of the sound field is not broad enough in width to generate higher levels of energy transfer for this order. The power coupled into the cell in the +1 order, $\psi_{+1}$, is physically coupled to the higher $\psi_{+2}$, but this order is not being included in the formalism. This raises the question of how physically accurate is the solution presented in Fig. 4 . To answer this Eq. (32d) is repeated now with a comparison to a 10-order numerical solution. The

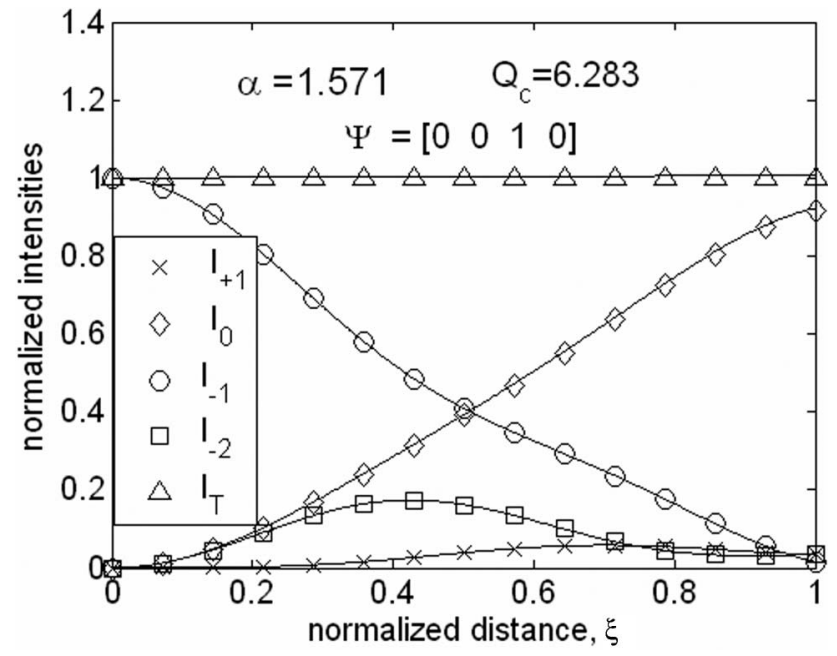

Fig. 4. Comparison for four-order numerical and analytical solutions; all power is initially in the -1 order. 
10-order numerical solution is calculated from Eq. (15) by limiting the values of $n$ for the range $-5 \leq n \leq 4$. To limit distractions only the numerical results for orders $\{-2,-1,0,+1\}$ are shown in Fig. $\underline{6}$. For the conditions taken, the 10-order numerical solution deviates in the $\psi_{-2}(\xi)$ by at most $10 \%$ while the other orders are essentially indistinguishable from those found in Fig. 5 after permutation in Eq. (40). For the $\left(\alpha, Q_{c}\right)$ conditions, the analytical solution is a reasonable approximation with no more than $10 \%$ error. It is expected that if either the sound intensity is increased or the Klein-Cook parameter is decreased the observed discrepancy will become higher.

A demonstration of the robustness of the solution and its ability to accommodate a general combination of initial conditions was performed. Specific initial conditions for this test put half of the power in the 0 th order and half of the power in the -1 order. As can be seen in Fig. 7 the four-order analytical (symbols) and the four-order numerical (solid line) predictions are in agreement. Due to physical symmetry for this test case created by the symmetry in the initial conditions it is expected that both the 0th order and the -1 order will overlay, and this can be confirmed from Fig. 7. This is also true, from the same symmetry argument, for the +1 order and the -2 order. Note that, consistent with physical considerations, the exchange of energy is symmetric and less than $10 \%$ of the total energy is transferred from the Bragg regime combination of 0 -order and -1 order to the outer pair combination of the +1 order and -2 order. The assumption being made, Fig. 7, is that the two incident beams, 0 th order and -1 order, are coherently related to the zero phase offset. If the two incident beams are phase incoherent then the appropriate method of solving would be to apply either analytical or numerical formalism for each incident light beam separately to predict the corresponding intensities at the output. Specifically, scale the intensities shown in Figs. 3 and $\underline{4}$ by $1 / 2$. Then, according to the principle of

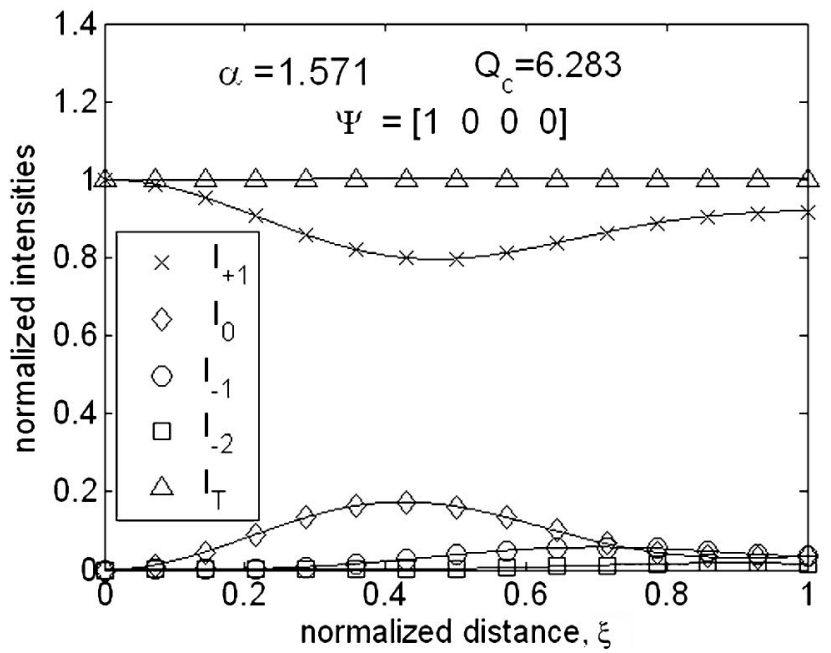

Fig. 5. Comparison for four-order numerical and analytical solutions; all power is initially in the +1 order.

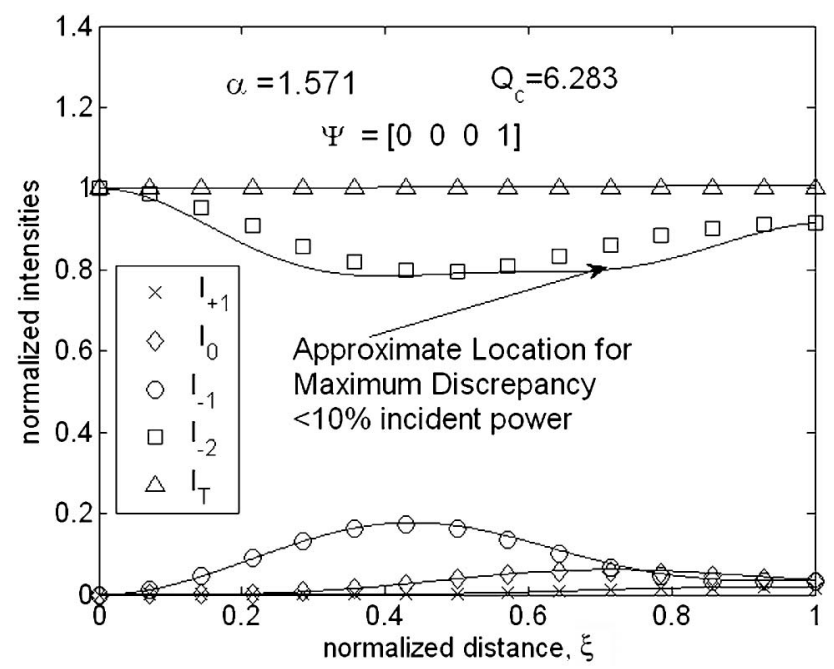

Fig. 6. Comparison for 10-order numerical and analytical solutions; all power is initially in the -2 order. The arrow designates the approximate location of the maximum discrepancy between numerical and analytical curves, estimated to be less than $10 \%$ of incident power.

superposition of intensities for incoherent light, add the results.

\section{Concluding Remarks}

An exact four-order solution with incident light conditions corresponding to any of the four diffracted orders has been presented. The solution was restricted by requiring that the angle for the 0th order be fixed and equal to the Bragg angle at the center frequency of the AO transducer. As demonstrated, the solution can be cast into the form of a transition matrix. Energy conservation for the four-order analytical solution was proved by first showing that the transition matrix is unitary. Second, it was shown that the unitary matrix condition is a sufficient condition for the energy conservation rule applicable for all orders considered. Comparisons of the analytical solution

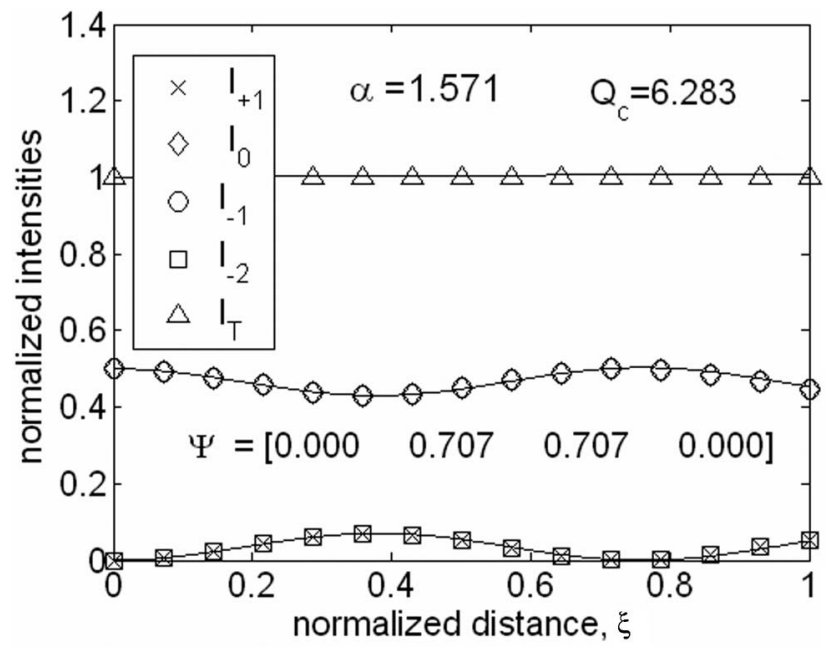

Fig. 7. Comparison for four-order numerical and analytical solutions; power is initially $50 \%$ in the 0th order and $50 \%$ in the -1 order. 


$$
|A(\xi)-\lambda I|=\left|\begin{array}{cccc}
-\lambda & \exp \left(-j Q_{c} \xi\right) & 0 & 0 \\
\exp \left(+j Q_{c} \xi\right) & -\lambda & 1 & 0 \\
0 & 1 & -\lambda & \exp \left(+j Q_{c} \xi\right) \\
0 & 0 & \exp \left(-j Q_{c} \xi\right) & -\lambda
\end{array}\right|
$$

and numerically generated solutions in four different cases were used to validate the analytical solution. The physical limitations in the usage and accuracy of the four-order analytical solution were discussed in Section $\underline{4}$, and it is believed that most of these restrictions can be removed with future work. In a recent investigation a dual input hybrid AO flip-flop was proposed and its performance analyzed using a strong two-order model for strong AO interaction [14]. Due the element of chaotic oscillation in their calculations, we believe that our four-order quadinput strong interaction solution developed here could be used to refine the analysis by more accurately accounting for the $\left\{I_{-2}, I_{+1}\right\}$ orders. Finally, it was found that we can now include acousto-optics in the long list of physical, mathematical, and structural examples for which the ubiquitous Euclid's Divine Proportion appears.

\section{Appendix A: Divine Proportion in Acousto-Optics}

It can be recognized that Eq. (17) is a linear homogeneous first-order system of differential equations. If the system of differential equations also had constant coefficients, i.e., $A$ is a constant matrix, it would be possible to predict the state transition matrix from [13]

$$
G\left(\xi, \xi_{o}\right)=\exp \left(A\left(\xi-\xi_{o}\right)\right)
$$

where

$$
\Psi(\xi)=\mathrm{G}\left(\xi, \xi_{o}\right) \cdot \Psi\left(\xi_{o}\right),
$$

and $\Psi\left(\xi_{o}\right)$ is the known initial conditions at some specified location $\xi_{o}$. Expanding the exponential of matrix (A1) into four additive matrix terms using the eigenvalues of $A$ is then possible using Sylvester's theorem [15]. However, because $A$ is not a constant matrix the (A1) approach is not applicable. Nonetheless it was discovered that the matrix operator for $A$, taken from Eq. (17), did have four distinct eigenvalues, all of which are related as shown below to Euclid's Divine Proportion also known as the Golden Ratio. Here we demonstrate the appearance of the Divine Proportion constant $\Phi$ in the AO problem considered. Euclid's Divine Proportion is associated with the numerical constant [10]

$$
\Phi=\frac{1+\sqrt{5}}{2} \approx 1.61
$$

To find the eigenvalues from Eq. (18), one method is to evaluate the determinant which leads to a quartic polynomial equation in $\lambda$ :

$$
\lambda^{4}-3 \lambda^{2}+1=0,
$$

which is biquadratic in $\lambda^{2}$. After observing that

$$
\frac{3+\sqrt{5}}{2}=\left(\frac{1+\sqrt{5}}{2}\right)^{2}, \quad \frac{3-\sqrt{5}}{2}=\frac{2}{3+\sqrt{5}},
$$

it follows that the four roots of biquadratic equation (A4) are

$$
\pm \sqrt{\frac{3+\sqrt{5}}{2}}= \pm \Phi, \quad \pm \sqrt{\frac{3-\sqrt{5}}{2}}= \pm \frac{1}{\Phi} .
$$

\section{Appendix B: Obtaining the Fourth-Order Differential Equation}

From Eq. (15), we write a system of differential equations as follows:

$$
\frac{d \psi_{+1}}{d \xi}=-j \alpha e^{-j Q_{c} \xi} \psi_{0}
$$

$$
\frac{d \psi_{0}}{d \xi}=-j \alpha e^{j Q_{c} \xi} \psi_{+1}-j \alpha \psi_{-1}
$$

$$
\begin{gathered}
\frac{d \psi_{-1}}{d \xi}=-j \alpha e^{j Q_{c} \xi} \psi_{-2}-j \alpha \psi_{0}, \\
\frac{d \psi_{-2}}{d \xi}=-j \alpha e^{-j Q_{c} \xi} \psi_{-1} .
\end{gathered}
$$

Using Eqs. (B1) and (푸), we solve for $\psi_{0}$ and $\psi_{-1}$ to get

$$
\psi_{0}=\frac{j e^{j Q_{c} \xi}}{\alpha} \frac{d \psi_{+1}}{d \xi}
$$

$$
\psi_{-1}=\frac{j e^{j Q_{c} \xi}}{\alpha} \frac{d \psi_{-2}}{d \xi} .
$$

Backsubstitution of Eqs. (B5) and (B6) into Eq. (B2) leads to the 2nd-order differential equation given by 


$$
\frac{d^{2} \psi_{+1}}{d \xi^{2}}+j Q_{c} \frac{d \psi_{+1}}{d \xi}+\alpha^{2} \psi_{+1}=-j \alpha \frac{d \psi_{-2}}{d \xi} .
$$

Similarly, backsubstitution of Eqs. (B5) and (B6) into Eq. (B3) leads to

$$
\frac{d^{2} \psi_{-2}}{d \xi^{2}}+j Q_{c} \frac{d \psi_{-2}}{d \xi}+\alpha^{2} \psi_{-2}=-j \alpha \frac{d \psi_{+1}}{d \xi} .
$$

After one differentiation of Eq. (B7), we have

$$
\frac{d^{3} \psi_{+1}}{d \xi^{3}}+j Q_{c} \frac{d^{2} \psi_{+1}}{d \xi^{2}}+\alpha^{2} \frac{d \psi_{+1}}{d \xi}=-j \alpha \frac{d^{2} \psi_{-2}}{d \xi^{2}} .
$$

After substitution of the right-hand side of Eq. (B8) into Eq. (B9), it leads to the 4th-order ordinary differential equation; Eq (19) in the main text.

\section{References}

1. P. Debye and F. W. Sears, "On the scattering of light by supersonic waves," Proc. Natl. Acad. Sci. USA 18, 409-414 (1932).

2. A. Korpel, Acousto-Optics (Marcel Dekker, 1988).

3. R. J. Pieper, A. Korpel, and W. Hereman, "Extension of the acoustic-optic Bragg regime through Hamming apodization of the sound field," J. Opt. Soc. Am. A 3, 1608-1619 (1986).
4. R. Pieper and A. Korpel, "A comparison of phased-array Bragg cells operating in the second order," Appl. Opt. 23, 2921-2934 (1984).

5. T.-C. Poon and A. Korpel, "Feynman diagram approach to acousto-optic scattering in the near-Bragg region," J. Opt. Soc. Am. 71, 1202-1208 (1981).

6. T.-C. Poon, "A Feynman diagram approach to multiple plane wave scattering in acousto-optic interactions," Ph.D. dissertation (University of Iowa, 1982), pp. 64-68.

7. E. Blomme and O. Leroy, "Diffraction of light by ultrasound at oblique incidence; An exact 4-order solution," Acustica 59, 182-192 (1986).

8. R. J. Pieper and A. Korpel, "A matrix formalism for the analysis of acousto-optic beam steering," Appl. Opt. 22, 4073-4081 (1983).

9. A. Korpel, "Two-dimensional plane wave theory of strong acousto-optic interaction in isotropic media," J. Opt. Soc. Am. 69, 678-683 (1979).

10. E. D. Nering, Elementary Linear Algebra (Saunders, 1974), p. 297.

11. P. Hemenway, Divine Proportion $\Phi($ Phi) in Art, Nature and Science (Stering, 2005), p. 14.

12. W. R. Klein and B. D. Cook, "Unified approach to ultrasonic light diffraction," IEEE Trans. Sonics Ultrason. SU-14, 123-133 (1967).

13. P. M. DeRusso, R. J. Roy, C. M. Close, and A. A. Desrochers, State Variables for Engineers, 2nd ed. (Wiley, 1966), p. 356.

14. S.-T. Chen and M. R. Chatterjee, "Dual-input acousto-optic set reset flip-flop and its nonlinear dynamics," Appl. Opt. 36, 3147-3154 (1997).

15. P. M. De Russo, R. J. Roy, C. M. Close, and A. A. Desrochers, State Variables for Engineers, 2nd ed. (Wiley, 1966), pp. 276-281. 